\title{
Analysis of the dynamical characteristics of fractional-order chaotic system and its DSP realization
}

\author{
Xintong Han, Junqiao Liu*, Huizhen Yan \\ \{dalianliujunqiao@126.com\} \\ School of Information Science and Engineering, Dalian polytechnic University, Dalian, 116034, China
}

\begin{abstract}
In this paper, the 4D fractional-order chaotic system is devised, and the accurate numerical solution of the system is obtained based on the CADM algorithm. The dynamical characteristics of the fractional-order chaotic system are analyzed by employing bifurcation diagram, Lyapunov exponential spectrum, 0-1 test and the poincaré section. Meanwhile, the ranges of parameters of the fractional-order chaotic system are given in practical application through the SE and $\mathrm{C} 0$ complexity algorithm. The results demonstrate that the fractional-order chaotic system with the complex dynamical behaviors. Finally, digital circuit of the chaotic system is implemented on the DSP platform. This study offers a practical and theoretical basis for the application of fractional-order chaotic system in the safe communication and other fields.
\end{abstract}

Keywords: fractional-order system, dynamical characteristics, the CADM algorithm, DSP implementation

\section{Introduction}

In 1695, Leibniz and Lopida proposed the concept of fractional calculus, but for a long time, its practical application background has not been discovered, leading to slow progress in the related research of fractional calculus. However, recent studies have shown that, compared to integer-order calculus, the fractional-order calculus could better describe the objective physical world [1]. Therefore, the research on fractional calculus has become a hot spot. Research on the Chen system [2], Liu system [3] and simplified Lorenz system [4] and other nonlinear chaotic systems found that, compared to integer-order chaotic systems, fractional-order chaotic systems have more complex dynamical characteristics [5-7].

Currently, the solving algorithms of the fractional-order chaotic systems mainly include frequency domain method (FDM) [8], predictive correction method (ABM) [9] and Adomian 
decomposition method (ADM) [10]. These algorithms generally use the Riemann-Liuville definition and Caputo definition. But they all have some problems. First, they cannot satisfy some important properties of the fractional calculus [11], such as the product rule and chain rule. Second, the calculation process is very complicated [12]. Therefore, Khalil et al. proposed the new definition of the conformable fractional calculus [13]. The combination of the definition of the fractional calculus and the ADM algorithm can make up for the shortcomings of the existing algorithms for solving fractional differential equations [12, 14]. The CADM algorithm improves the ADM algorithm, reduces the complexity of calculation, and has the advantages of faster convergence speed, calculation speed, and less resource consumption [15]. At present, the use of this CADM algorithm to resolve the fractional-order chaotic systems is gradually becoming a research hotspot [16].

At present, many studies have been carried out on the application of fractional-order chaotic systems, such as nuclear magnetic resonance [17], cellular neural networks [18], and secure communications [19-21]. But it really applies to hardware and software technologies that rely on signal processing. Digital signal processor (DSP) $[22,23]$ has been widely used in engineering due to its superior performance and convenient processing. Based on this, this paper uses DSP technology to implement the hardware implementation of the proposed four-dimensional fractional-order chaotic system.

Based on the a four-dimensional Sprott-B chaotic system, the 4-D fractional-order chaotic system is constructed through using the conformable fractional calculus definition. The remaining structure of the paper is as follows. In sect.2, the numerical solution of the 4-D fractional-order chaotic system is solved by the CADM algorithm. The dynamical behaviors of the 4-D fractional-order chaotic system are analysed in sect.3. The 4-D fractional-order chaotic system is achieved on the DSP platform In sect.4. Finally, the experimental research results are summarized and a future direction is proposed.

\section{Numerical solution of the fractional-order chaotic system}

\subsection{CADM algorithm of the fractional-order chaotic system}

Assuming that the conformable fractional differential equation is expressed as

$$
\left\{\begin{array}{l}
T_{t}^{q} y(t)=L y(t)+N y(t)+g \\
y^{(k)}\left(t_{0}^{+}\right)=b_{k}, k=0, \cdots, m-1, \\
m \in N, m-1<q \leq m
\end{array}\right.
$$


where $T_{\mathrm{t}}^{\varphi}$ is differential operator, $x(\mathrm{t})=\left[y_{1}(t) y_{2}(t) \ldots y_{\mathrm{n}}(t)\right]^{\mathrm{T}}$ are the state variables. $L x(t)$ is the linear part of the system, $N y(t)$ is the non-linear part of the system function. $g$ represents the constant term, and $b_{\mathrm{k}}$ represents the initial value of the chaotic system. According to the conformable fractional calculus definition, one can get

$$
y(t)=I_{t_{0}}^{q} L y(t)+I_{t_{0}}^{q} N y(t)+I_{t_{0}}^{q} g+y\left(t_{0}\right),
$$

here $I_{t_{0}}^{q}$ is the integral operator, $y\left(t_{0}\right)$ is the initial value condition of the chaotic system. The nonlinear term can be expressed as

$$
N y=\sum_{i=0}^{\infty} A^{i}\left(y^{0}, y^{1}, \cdots, y^{i}\right),
$$

then the nonlinear term can be decomposed by

$$
\left\{\begin{array}{l}
A_{j}^{i}=\frac{1}{i !}\left[\frac{d^{i}}{d \lambda^{i}} N\left(v_{j}^{i}(\lambda)\right)\right]_{\lambda=0}, \\
v_{j}^{i}(\lambda)=\sum_{k=0}^{i}(\lambda)^{k} y_{j}^{k}
\end{array},\right.
$$

where $i=0,1, \ldots, j=1,2, \ldots, \mathrm{n}$. So, the numerical solution of the equation is

$$
y=\sum_{i=0}^{\infty} y^{i}=I_{t 0}^{q} L \sum_{i=0}^{\infty} y^{i}+I_{t 0}^{q} \sum_{i=0}^{\infty} A^{i}+I_{t 0}^{q} \mathrm{~g}+y\left(t_{0}\right),
$$

here $y_{i}$ is calculated by

$$
\left\{\begin{array}{l}
y^{0}=I_{t_{0}}^{q} g+y\left(t_{0}\right) \\
y^{1}=I_{t_{0}}^{q} L y^{0}+I_{t_{0}}^{q} A^{0}\left(y^{0}\right) \\
y^{2}=I_{t_{0}}^{q} L y^{1}+I_{t_{0}}^{q} A^{1}\left(y^{0}, y^{1}\right) \\
\cdots \\
y^{i}=I_{t_{0}}^{q} L y^{i-1}+I_{t_{0}}^{q} A^{i-1}\left(y^{0}, y^{1}, y^{2}, \cdots, y^{i-1}\right) \\
\cdots
\end{array} .\right.
$$

\subsection{Solution of the fractional-order chaotic system}

A new four-dimensional autonomous chaotic system on the basis of the proposed Sprott-B system. The new system equation can be expressed as

$$
\left\{\begin{array}{l}
\dot{x}_{1}=a\left(x_{2}-x_{1}\right)+x_{4} \\
\dot{x}_{2}=x_{1} x_{3} \\
\dot{x}_{3}=b-x_{1} x_{2} \\
\dot{x}_{4}=-c x_{4}+x_{1} x_{3}
\end{array},\right.
$$


here $x_{1}, x_{2}, x_{3}, x_{4}$ indicates the state variables of the system, $a, b$ and $c$ are positive parameters of the system. Based on conformable derivative, the fractional form of the chaotic system equation is

$$
\left\{\begin{array}{l}
T_{t}^{q} x_{1}=a\left(x_{2}-x_{1}\right)-x_{4} \\
T_{t}^{q} x_{2}=x_{1} x_{3} \\
T_{t}^{q} x_{3}=b-x_{1} x_{2} \\
T_{t}^{q} x_{4}=-c x_{4}+x_{1} x_{3}
\end{array}\right.
$$

here $q$ is the order of the system. According to the above formula (2), the linear, non-linear and constant terms are

$$
\left[\begin{array}{l}
L_{x_{1}} \\
L_{x_{2}} \\
L_{x_{3}} \\
L_{x_{4}}
\end{array}\right]=\left[\begin{array}{c}
a\left(x_{2}-x_{1}\right)+x_{4} \\
0 \\
0 \\
-c x_{4}
\end{array}\right],\left[\begin{array}{l}
N_{x_{1}} \\
N_{x_{2}} \\
N_{x_{3}} \\
N_{x_{4}}
\end{array}\right]=\left[\begin{array}{c}
0 \\
x_{1} x_{3} \\
-x_{1} x_{2} \\
x_{1} x_{3}
\end{array}\right],\left[\begin{array}{l}
g_{1} \\
g_{2} \\
g_{3} \\
g_{4}
\end{array}\right]=\left[\begin{array}{l}
0 \\
0 \\
\mathrm{~b} \\
0
\end{array}\right] .
$$

Decomposing the Eq. (6) the non-linear terms decompositions are

$$
\begin{aligned}
& \left\{\begin{array}{l}
A_{2}^{0}=x_{1}^{0} x_{3}^{0} \\
A_{2}^{1}=x_{1}^{1} x_{3}^{0}+x_{1}^{0} x_{3}^{1} \\
A_{2}^{2}=x_{1}^{2} x_{3}^{0}+x_{1}^{1} x_{3}^{1}+x_{1}^{0} x_{3}^{2} \\
A_{2}^{3}=x_{1}^{3} x_{3}^{0}+x_{1}^{2} x_{3}^{1}+x_{1}^{1} x_{3}^{2}+x_{1}^{0} x_{3}^{3} \\
A_{2}^{4}=x_{1}^{4} x_{3}^{0}+x_{1}^{3} x_{3}^{1}+x_{1}^{2} x_{3}^{2}+x_{1}^{1} x_{3}^{3}+x_{1}^{0} x_{3}^{4} \\
A_{2}^{5}=x_{1}^{5} x_{3}^{0}+x_{1}^{4} x_{3}^{1}+x_{1}^{3} x_{3}^{2}+x_{1}^{2} x_{3}^{3}+x_{1}^{1} x_{3}^{4}+x_{1}^{0} x_{3}^{5}
\end{array}\right. \\
& \left\{\begin{array}{l}
A_{3}^{0}=-x_{1}^{0} x_{2}^{0} \\
A_{3}^{1}=-x_{1}^{1} x_{2}^{0}-x_{1}^{0} x_{2}^{1} \\
A_{3}^{2}=-x_{1}^{2} x_{2}^{0}-x_{1}^{1} x_{2}^{1}-x_{1}^{0} x_{2}^{2} \\
A_{3}^{3}=-x_{1}^{3} x_{2}^{0}-x_{1}^{2} x_{2}^{1}-x_{1}^{1} x_{2}^{2}-x_{1}^{0} x_{2}^{3} \\
A_{3}^{4}=-x_{1}^{4} x_{2}^{0}-x_{1}^{3} x_{2}^{1}-x_{1}^{2} x_{2}^{2}-x_{1}^{1} x_{2}^{3}-x_{1}^{0} x_{2}^{4} \\
A_{3}^{5}=-x_{1}^{5} x_{2}^{0}-x_{1}^{4} x_{2}^{1}-x_{1}^{3} x_{2}^{2}-x_{1}^{2} x_{2}^{3}-x_{1}^{1} x_{2}^{4}-x_{1}^{0} x_{2}^{5}
\end{array}\right.
\end{aligned}
$$




$$
\left\{\begin{array}{l}
A_{4}^{0}=x_{1}^{0} x_{3}^{0} \\
A_{4}^{1}=x_{1}^{1} x_{3}^{0}+x_{1}^{0} x_{3}^{1} \\
A_{4}^{2}=x_{1}^{2} x_{3}^{0}+x_{1}^{1} x_{3}^{1}+x_{1}^{0} x_{3}^{2} \\
A_{4}^{3}=x_{1}^{3} x_{3}^{0}+x_{1}^{2} x_{3}^{1}+x_{1}^{1} x_{3}^{2}+x_{1}^{0} x_{3}^{3} \\
A_{4}^{4}=x_{1}^{4} x_{3}^{0}+x_{1}^{3} x_{3}^{1}+x_{1}^{2} x_{3}^{2}+x_{1}^{1} x_{3}^{3}+x_{1}^{0} x_{3}^{4} \\
A_{4}^{5}=x_{1}^{5} x_{3}^{0}+x_{1}^{4} x_{3}^{1}+x_{1}^{3} x_{3}^{2}+x_{1}^{2} x_{3}^{3}+x_{1}^{1} x_{3}^{4}+x_{1}^{0} x_{3}^{5}
\end{array} .\right.
$$

here, assuming the initial value $x_{0}=\left[x_{1}(0), x_{2}(0), x_{3}(0), x_{4}(0)\right]$, the first term can be described as

$$
\left\{\begin{array}{l}
x_{1}^{0}=x_{1}\left(t_{0}\right) \\
x_{2}^{0}=x_{2}\left(t_{0}\right) \\
x_{3}^{0}=x_{3}\left(t_{0}\right)+b \frac{h^{q}}{q} \\
x_{4}^{0}=x_{4}\left(t_{0}\right)
\end{array} .\right.
$$

Let

$$
\left\{\begin{array}{l}
c_{1}^{0}=x_{1}^{0} \\
c_{2}^{0}=x_{2}^{0} \\
c_{3}^{0}=x_{3}^{0} \\
c_{4}^{0}=x_{4}^{0}
\end{array}\right.
$$

according to the Eq. (6), the second expansion is

$$
\left\{\begin{array}{l}
x_{1}^{1}=\left[a\left(c_{2}^{0}-c_{1}^{0}\right)+c_{4}^{0}\right] \frac{\left(t-t_{0}\right)^{q}}{q} \\
x_{2}^{1}=\left[c_{1}^{0} c_{3}^{0}\right] \frac{\left(t-t_{0}\right)^{q}}{q} \\
x_{3}^{1}=\left[-c_{1}^{0} c_{2}^{0}\right] \frac{\left(t-t_{0}\right)^{q}}{q} \\
x_{4}^{1}=\left[-c c_{4}^{0}+c_{1}^{0} c_{3}^{0}\right] \frac{\left(t-t_{0}\right)^{q}}{q}
\end{array} .\right.
$$

Let $h=t-t_{0}$, by the same method, the coefficients of the other five terms are

$$
\left\{\begin{array}{l}
c_{1}^{2}=a\left(c_{2}^{1}-c_{1}^{1}\right)+c_{4}^{1} \\
c_{2}^{2}=c_{1}^{1} c_{3}^{0}+c_{1}^{0} c_{3}^{1} \\
c_{3}^{2}=-c_{1}^{1} c_{2}^{0}-c_{1}^{0} c_{2}^{1} \\
c_{4}^{2}=-c c_{4}^{1}+c_{1}^{1} c_{3}^{0}+c_{1}^{0} c_{3}^{1}
\end{array},\right.
$$




$$
\begin{gathered}
\left\{\begin{array}{l}
c_{1}^{3}=a\left(c_{2}^{2}-c_{1}^{2}\right)+c_{4}^{2} \\
c_{2}^{3}=c_{1}^{2} c_{3}^{0}+2 c_{1}^{1} c_{3}^{1}+c_{1}^{0} c_{3}^{2} \\
c_{3}^{3}=-c_{1}^{2} c_{2}^{0}-2 c_{1}^{1} c_{2}^{1}-c_{1}^{0} c_{2}^{2} \\
c_{4}^{3}=-c c_{4}^{2}+c_{1}^{2} c_{3}^{0}+2 c_{1}^{1} c_{3}^{1}+c_{1}^{0} c_{3}^{2}
\end{array}\right. \\
\left\{\begin{array}{l}
c_{1}^{4}=a\left(c_{2}^{3}-c_{1}^{3}\right)+c_{4}^{3} \\
c_{2}^{4}=c_{1}^{3} c_{3}^{0}+3 c_{1}^{2} c_{3}^{1}+3 c_{1}^{2} c_{3}^{1}+c_{1}^{0} c_{3}^{3} \\
c_{3}^{4}=-c_{1}^{3} c_{2}^{0}-3 c_{1}^{2} c_{2}^{1}-3 c_{1}^{1} c_{2}^{2}-c_{1}^{0} c_{2}^{3} \\
c_{4}^{4}=-c c_{4}^{3}+c_{1}^{3} c_{3}^{0}+3 c_{1}^{2} c_{3}^{1}+3 c_{1}^{2} c_{3}^{1}+c_{1}^{0} c_{3}^{3}
\end{array}\right. \\
\left\{\begin{array}{l}
c_{1}^{5}=a\left(c_{2}^{4}-c_{1}^{4}\right)+c_{4}^{4} \\
c_{2}^{5}=c_{1}^{4} c_{3}^{0}+4 c_{1}^{3} c_{3}^{1}+6 c_{1}^{2} c_{3}^{2}+4 c_{1}^{1} c_{3}^{3}+c_{1}^{0} c_{3}^{4} \\
c_{3}^{5}=-c_{1}^{4} c_{2}^{0}-4 c_{1}^{3} c_{2}^{1}-6 c_{1}^{2} c_{2}^{2}-4 c_{1}^{1} c_{2}^{3}-c_{1}^{0} c_{2}^{4} \\
c_{4}^{5}=-c c_{4}^{4}+c_{1}^{4} c_{3}^{0}+4 c_{1}^{3} c_{3}^{1}+6 c_{1}^{2} c_{3}^{2}+4 c_{1}^{1} c_{3}^{3}+c_{1}^{0} c_{3}^{4}
\end{array}\right. \\
\left\{\begin{array}{l}
c_{1}^{6}=a\left(c_{2}^{5}-c_{1}^{5}\right)+c_{4}^{5} \\
c_{2}^{6}=c_{1}^{5} c_{3}^{0}+5 c_{1}^{4} c_{3}^{1}+10 c_{1}^{3} c_{3}^{2}+10 c_{1}^{2} c_{3}^{3}+5 c_{1}^{1} c_{3}^{4}+c_{1}^{0} c_{3}^{5} \\
c_{3}^{6}=-c_{1}^{5} c_{2}^{0}-5 c_{1}^{4} c_{2}^{1}-10 c_{1}^{3} c_{2}^{2}-10 c_{1}^{2} c_{2}^{3}-5 c_{1}^{1} c_{2}^{4}-c_{1}^{0} c_{2}^{5} \\
c_{4}^{6}=-c c_{4}^{5}+c_{1}^{5} c_{3}^{0}+5 c_{1}^{4} c_{3}^{1}+10 c_{1}^{3} c_{3}^{2}+10 c_{1}^{2} c_{3}^{3}+5 c_{1}^{1} c_{3}^{4}+c_{1}^{0} c_{3}^{5}
\end{array}\right.
\end{gathered}
$$

so, the CADM numerical results of the fractional-order chaotic system with six terms are

$$
x_{j}(t)=\sum_{i=0}^{6} c_{j}^{i} \frac{\left(t-t_{0}\right)^{i q}}{i ! q^{i}} .
$$

\section{Dynamical analysis of the fractional-order chaotic system}

\subsection{Simulation analysis of the system}

Setting $a=4, b=9, c=5, h=0.01$, the system order $q=0.8$, and the initial values $x_{0}=[1,1,1,1]$. The Lyapunov exponents of the system are $L_{1}=1.0170, L_{2}=0, L_{3}=-12.5479$, and $L_{4}=-16.7206$, and the Lyapunov dimension is $D_{\mathrm{L}}=2.09$. At this time, the system has a positive Lyapunov exponent, indicating that the system is chaotic. Fig. 1 shows the phase diagrams in different planes of the system. To further verify the dynamical characteristics of the system under the parameters, the Poincaré section of the system at this time are shown in Fig. 2. The Poincaré section in the figure is neither a finite point set nor a closed curve. It is a piece of dense points with a fractal structure. This structure has the typical characteristics of a chaotic system. 


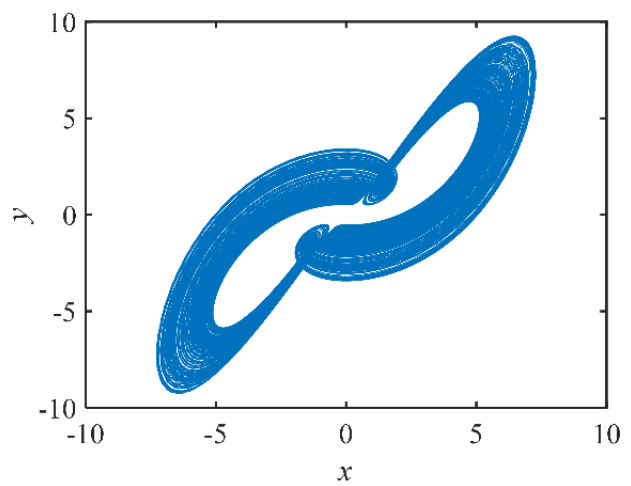

(a)

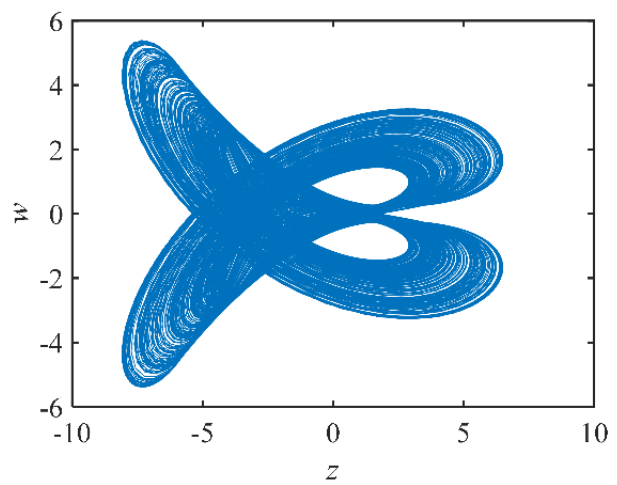

(b)

Fig. 1 The phase diagrams of system with $q=0.8$

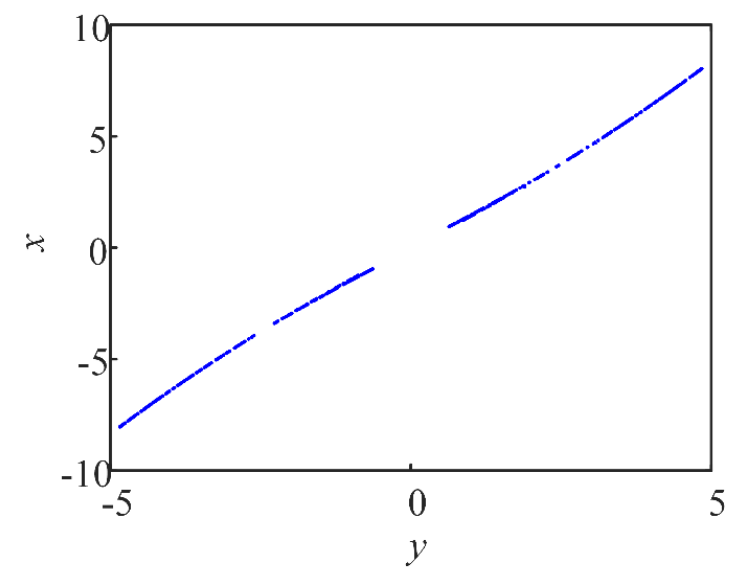

Fig. 2 The Poincaré section of system (8) on the $y$ - $x$ plane when $q=0.8$

\subsection{Stability analysis of the system}

For Eq. (7), let $\dot{x}_{1}=x_{2}=x_{3}=x_{4}=0$, one can get the equilibrium point of the system $S_{1,2}=( \pm \sqrt{ } \mathrm{b}, \pm$ $\sqrt{ } \mathrm{b}, 0,0)$. The Jacobian matrix $J$ for the system (Eq. (7)) at the plane equilibrium $S_{1,2}$ is obtained as

$$
J=\left(\begin{array}{cccc}
-a & a & 0 & 1 \\
0 & 0 & \pm \sqrt{b} & 0 \\
\mp \sqrt{b} & \mp \sqrt{b} & 0 & 0 \\
0 & 0 & \pm \sqrt{b} & -c
\end{array}\right)
$$

when $a=4, b=9$, and $c=5$, the equilibrium point of the system is $S_{1,2}=( \pm 3, \pm 3,0,0)$. The 
characteristic polynomial equation is yielded as

$$
\lambda^{32}+9 \lambda^{24}+29 \lambda^{16}+126 \lambda^{8}+360=0 .
$$

If the eigenvalue satisfies

$$
|\arg (\lambda)| \geq \frac{\pi}{2 M},
$$

the system is gradually stable at the equilibrium point. Substitute eigenvalues into the Eq. (24),

$$
0.05 \pi-\min _{i}\left\{\left|\arg \left(\lambda_{i}\right)\right|\right\}=-0.0229<0,
$$

thus, at the equilibrium point $S_{1,2}$, the system is stable.

\subsection{Dynamical characteristics with the parameter varying}

\subsubsection{Dynamical evolution with parameter $a$}

Setting the parameters $b=9, c=5$, the order $q=0.8, h=0.01$, and $x_{0}=\left[\begin{array}{llll}1 & 1 & 1 & 1\end{array}\right]$. Fig. 3 indicates the bifurcation diagrams and the Lyapunov exponents spectrum when $a$ changes from1 to 5 . When $a$ belong to [2.98,3.03], [3.46,3.5], [4.27,4.33], [4.34,5] and so on, the largest Lyapunov exponent of the system is zero, so when the parameter $a$ is within these ranges, the system is in the periodic state. However, in other ranges, the largest Lyapunov exponent is positive, and the system is in the chaotic state. Complex dynamical characteristics of the system with the parameter $a$ varying are summarized in the Table 1 .

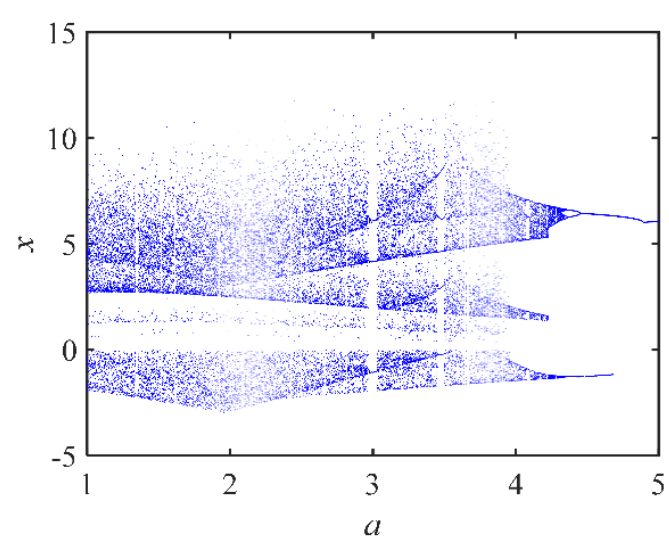

(a)

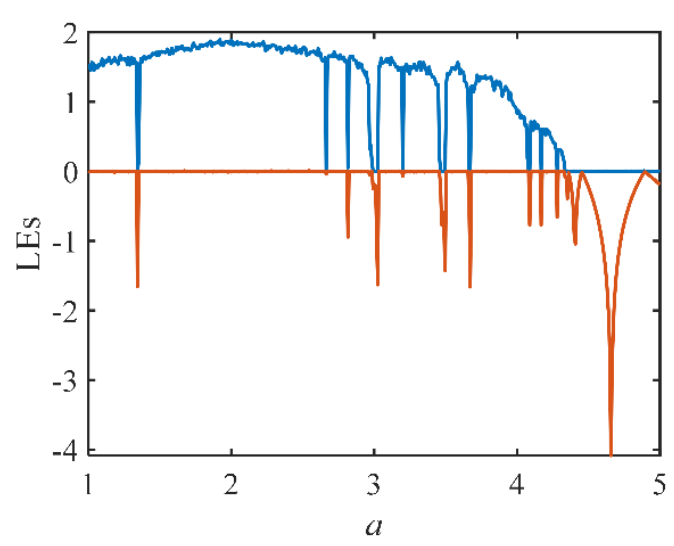

(b)

Fig. 3 The system varies with the parameter $a$ (a)Lyapunov exponent spectrum; (b) Bifurcation diagram. 
Table 1. System status with the parameter $a$

\begin{tabular}{cccc}
\hline$a$ & dynamical characteristic & $a$ & $\begin{array}{c}\text { dynamical } \\
\text { characteristic }\end{array}$ \\
\hline $1 \sim 2.97$ & Chaotic attractor & $4.18 \sim 4.20$ & Chaotic attractor \\
$2.98 \sim 3.03$ & Period-2 state & $4.21 \sim 4.22$ & Period-7 state \\
$3.04 \sim 3.45$ & Chaotic attractor & $4.23 \sim 4.27$ & Chaotic attractor \\
$3.46 \sim 3.5$ & Period-3 state & $4.28 \sim 4.33$ & Period-3 state \\
$3.51 \sim 3.65$ & Chaotic attractor & $4.33 \sim 4.34$ & Chaotic attractor \\
$3.66 \sim 3.67$ & Period-2 state & $4.34 \sim 4.36$ & Period-4 state \\
$3.68 \sim 4.15$ & Chaotic attractor & $4.36 \sim 4.45$ & Period-2 state \\
$4.16 \sim 4.17$ & Period-5 state & $4.46 \sim 5$ & Limit cycle \\
\hline
\end{tabular}

From the Table 1, when $a$ from 1 to 5, a typical chaotic attractor and six different types of periodic states appear in the system. In the Fig.4, it can be found that when the chaotic system is in a periodic state, there is a bounded regular motion on the $p$-s plane; when the system is in the chaotic state, the $p-s$ plane is an unbounded motion similar to Brownian motion. 

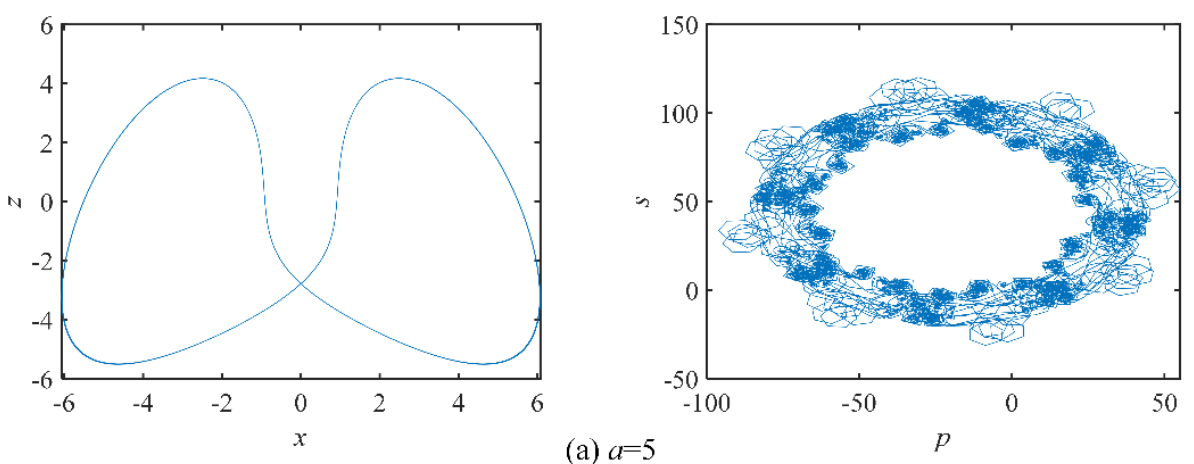

(a) $a=5$
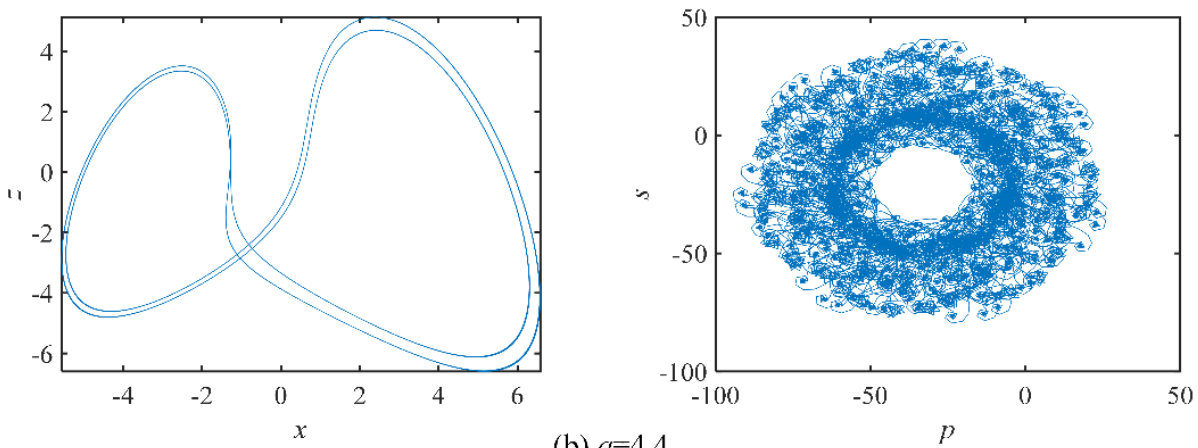

(b) $a=4.4$
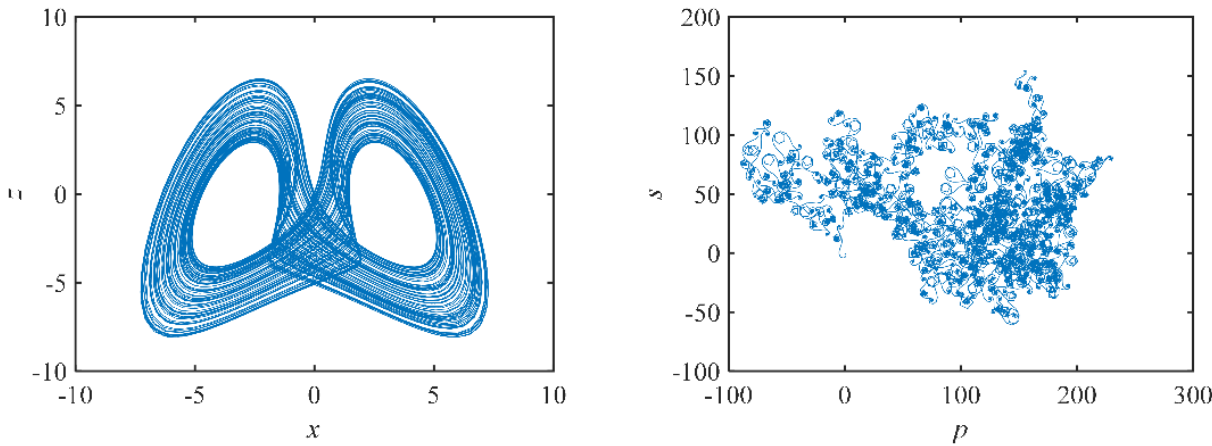

(c) $a=4$

Fig. 4 Phase diagram and $p$-s plane with different values of $a$.

\subsubsection{Dynamical evolution with the parameter $b$}

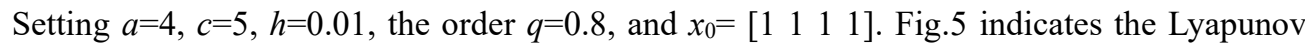
exponents spectrum and the bifurcation diagrams when the parameter $b$ varies in the range of $(7,11)$. The results can be found from the bifurcation diagram that as the parameter $b$ 
increases, the system enters the chaotic state from a periodic state through the period-doubling bifurcation. At the same time, when the parameter $b$ is at $[8.53,8.59]$ and $[10.21,10.25]$, there are two relatively obvious period window, which corresponds to the Lyapunov exponential spectrum. When the parameter $b$ varying, dynamical characteristics of the system are shown in the Table 2.

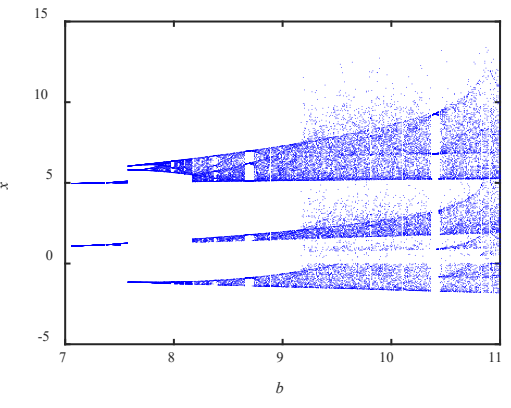

(a)

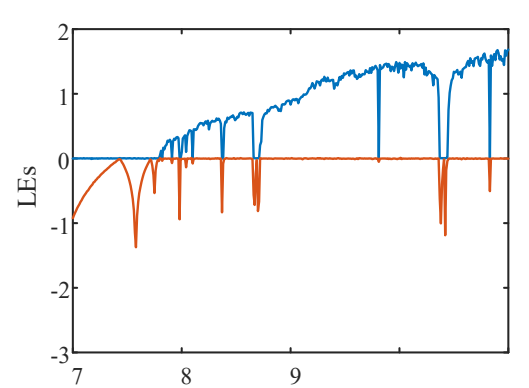

(b)

Fig. 5 The system with the parameter $b$ varies. (a) Lyapunov exponent spectrum

(b) Bifurcation diagram

Table 2. System status with the parameter $b$

\begin{tabular}{cccc}
\hline$b$ & $\begin{array}{c}\text { dynamical } \\
\text { characteristic }\end{array}$ & $b$ & $\begin{array}{c}\text { dynamical } \\
\text { characteristic }\end{array}$ \\
\hline $7 \sim 7.33$ & Limit cycle & $8.27 \sim 8.52$ & Chaotic attractor \\
$7.34 \sim 7.72$ & Period-2 state & $8.53 \sim 8.59$ & Period-3 state \\
$7.73 \sim 7.8$ & Period-4 state & $8.60 \sim 9.77$ & Chaotic attractor \\
$7.81 \sim 7.97$ & Chaotic attractor & $9.78 \sim 9.80$ & Period-3 state \\
$7.8 \sim 8.01$ & Period-3 state & $9.81 \sim 10.20$ & Chaotic attractor \\
$8.02 \sim 8.24$ & Chaotic attractor & $10.21 \sim 10.25$ & Period-2 state \\
$8.25 \sim 8.26$ & Period-5 state & $10.26 \sim 11$ & Chaotic attractor \\
\hline
\end{tabular}

\subsubsection{Dynamical evolution with the order $q$}

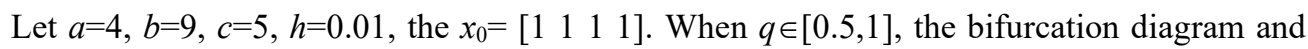
the Lyapunov exponents spectrum are shown in the Fig.6. From Fig.6(a), when $q \in[0.53,1]$, the system is in a chaotic state. When $q<0.52$, there is no value in the Lyapunov exponent spectrum and bifurcation diagram, and the chaotic system is in the divergent state. 


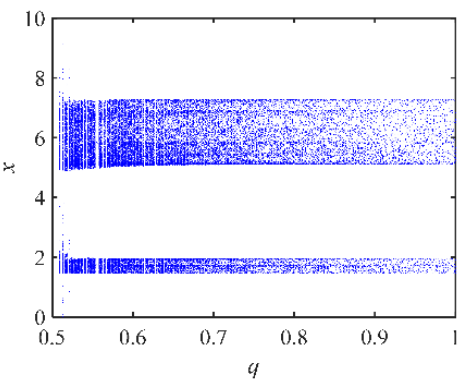

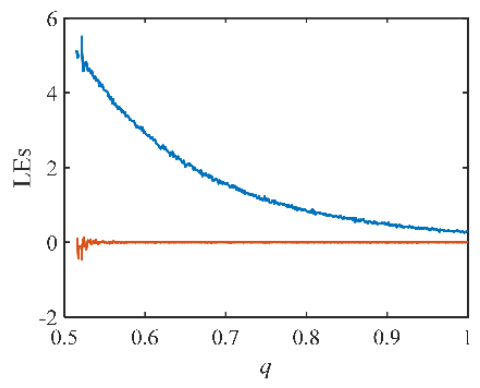

(b)

Fig. 6 The system with the parameter $q$ varies. (a) Lyapunov exponent spectrum (b)Bifurcation diagram

To further analyze the system is applicable to the field of secure communication parameter selection range, observing better dynamics chaotic system, where SE and C0 algorithm [24-26] of the system are analyzed. Let the order $q \in[0.5,1]$, sequence length $N=50000$, SE and C0 complexity of the system are shown in the Fig.7. When $q>0.52$, the change trend of SE complexity and $\mathrm{C} 0$ complexity is gradually reduced. When $q=1$, the value of complexity reaches the minimum. Therefore, the fractional-order chaotic system is more complicated than the integer-order chaotic system. As the order decreases, the system complexity will increase correspondingly, which indicates that the fractional-order state of the system has higher application value than the integer-order state.

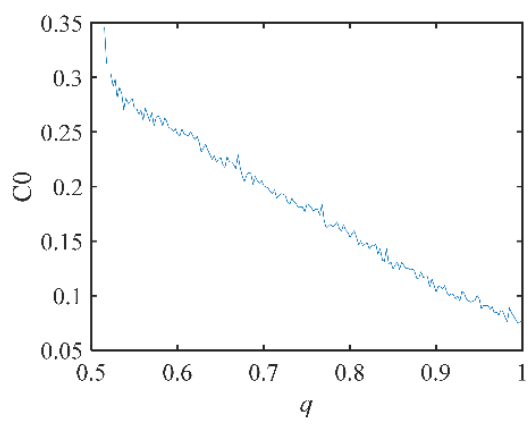

(a)

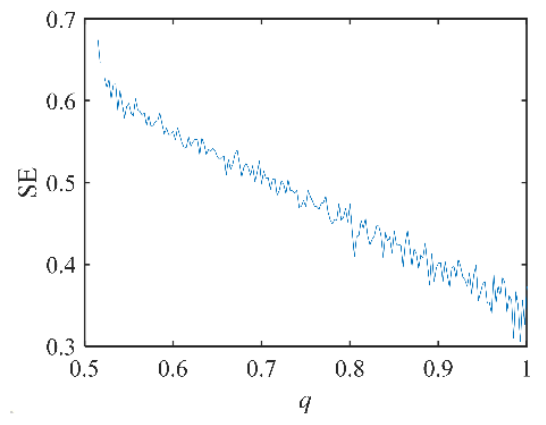

(b)

Fig. 7 Complexity varies with the parameter $q$ (a) C0 complexity (b)SE complexity.

\section{Hardware circuit based on DSP}

In this section, the digital circuit fractional chaotic systems are constructed and implemented on a DSP platform. DSP processor is widely used in engineering practice, due to its powerful performance and low price. Where, the processing speed of the DSP chip TMS320F28335 is accelerated, the precision is higher, and the reliability is higher. TMS320F28335 16-bit DSP is 
a kind of floating-point arithmetic operation at $150 \mathrm{MHz}$. The DSP board is connected to 16 pairs of interface channels by DAC DAC8552 SPI (Serial Peripheral Interface). The program flow for DSP implementation and the DSP experimental platform of the fractional-order chaotic system are shown in the Fig. 8 and Fig. 9. Setting $a=4, b=9, c=5, h=0.01, q=0.8$, and $x_{0}=\left[\begin{array}{llll}1 & 1 & 1 & 1\end{array}\right]$. The experimental results of the DSP platform are shown in the Fig. 10. Experimental results with the corresponding numerical results agree well in Fig. 1. That is to say, the 4-D fractional-order chaotic system has been perfectly tested on the DSP platform.

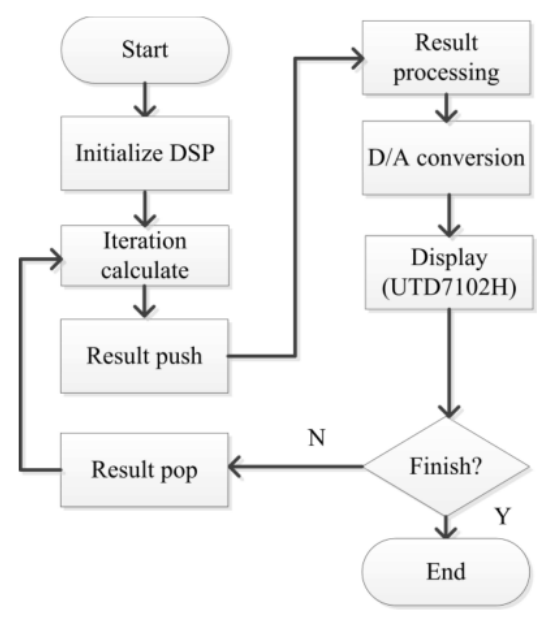

Fig. 8 The program flow for DSP implementation

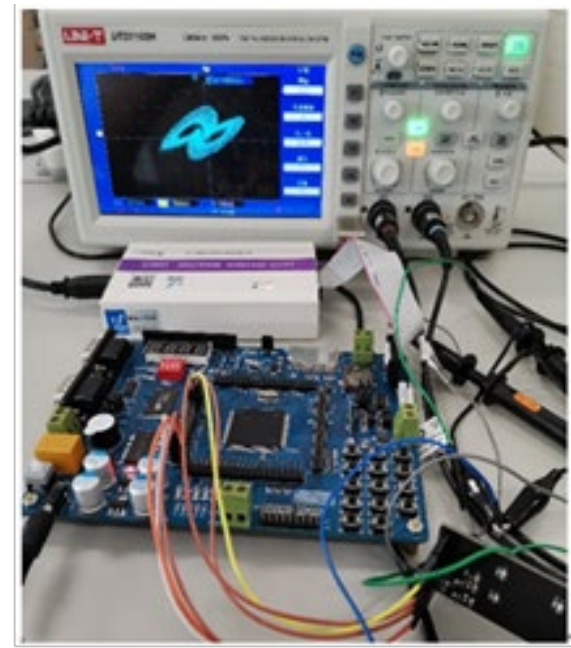

Fig. 9 The DSP experimental platform 

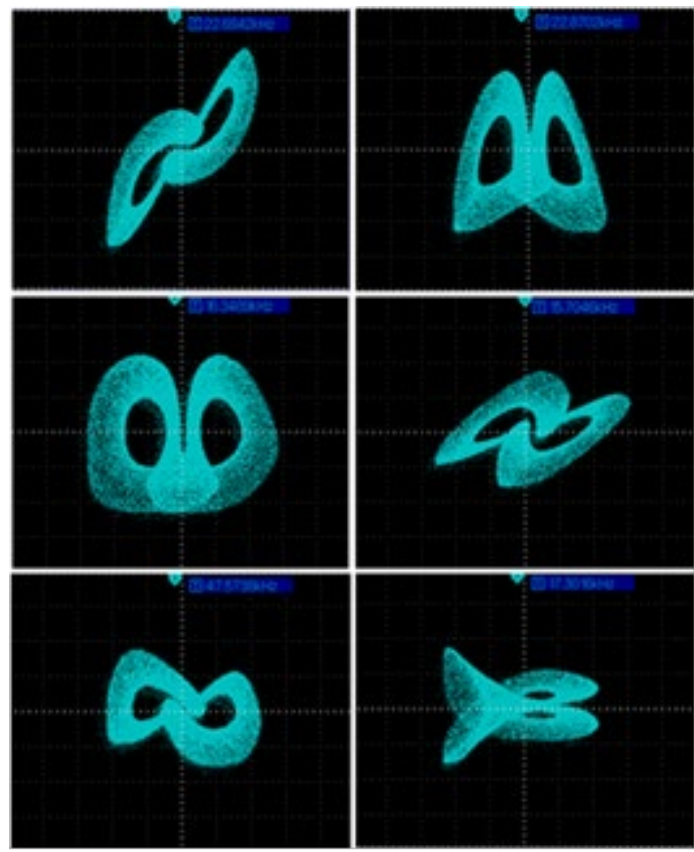

Fig. 10 The DSP implementation of the system

\section{Conclusion}

In this paper, the 4-D fractional-order chaotic system is designed. The numerical solutions of the fractional-order chaotic system are solved by using the CADM algorithm. Meanwhile, the dynamical behaviors of the system were analyzed through phase diagrams, bifurcation diagrams and Lyapunov exponents. The simulation results show that the fractional-order chaotic system has complex dynamics. The minimum order of chaos in the fractional-order chaotic system is 2.12 . In addition, the analysis of complexity shows that, when $q=0.53$, system complexity is the highest, the randomness of the chaotic sequence is the best and the safety performance is the highest. Finally, the system is implemented by using the DSP platform. The experimental results reflect the correctness of the CADM algorithm and the physical feasibility of the fractional-order chaotic system. The results for the system described herein provides a theoretical basis in the application of engineering.

\section{References}

1. Tavazoei, M.S., et al., Some Applications of Fractional Calculus in Suppression of Chaotic Oscillations. IEEE Transactions on Industrial Electronics, 2008. 55(11): p. 4094-4101.

2. A, C.L. and G.C. B, Chaos in the fractional order Chen system and its control. Chaos, Solitons 
\& Fractals, 2004. 22(3): p. 549-554.

3. Lu, J.J. and C.X. Liu, Realization of fractional-order Liu chaotic system by circuit. Chinese Physics B, 2007. 16(6): p. 1586.

4. Sun, K., X. Wang, and J.C. Sprott, Bifurcations and Chaos in fractional-Order Simplified Lorenz System. International Journal of Bifurcation \& Chaos, 2010. 20(04): p. 1209-1219.

5. Shaobo, et al., Solution and dynamics analysis of a fractional-order hyperchaotic system. Mathematical Methods in the Applied Sciences, 2016.

6. Zhang, L., et al., Solution and dynamics of a fractional-order 5-D hyperchaotic system with four wings. European Physical Journal Plus, 2017. 132(1): p. 31.

7. Yu, F., et al., Dynamic Analysis, Circuit Design, and Synchronization of a Novel $6 D$ Memristive Four-Wing Hyperchaotic System with Multiple Coexisting Attractors. Complexity, 2020. 2020.

8. Charef, A., et al., Fractal system as represented by singularity function. IEEE Transactions on Automatic Control, 2002. 37(9): p. 1465-1470.

9. Sun, H.H., A.A. Abdelwahab, and B. Onaral, Linear approximation of transfer function with a pole of fractional power. Automatic Control IEEE Transactions on, 1984. 29(5): p. 441-444.

10. Adomian, G., A review of the decomposition method and some recent results for nonlinear equations. Mathematical \& Computer Modelling, 1991. 13(7): p. 17-43.

11. He, S., et al., Numerical analysis of a fractional-order chaotic system based on conformable fractional-order derivative. European Physical Journal Plus, 2017. 132(1): p. 36.

12. Abdeljawad, T., On conformable fractional calculus. 2015: Elsevier Science Publishers B. V.

13. Khalil, R., et al., A new definition of fractional derivative. Journal of Computational and Applied Mathematics, 2014. 264(5): p. 65-70.

14. Gzütok, N.Y. and U. Gzütok, Multivariable Conformable Fractional Calculus. Filomat, 2018. 32(2): p. 45-53.

15. Allahviranloo, T., N. Ahmady, and E. Ahmady, Numerical solution of fuzzy differential equations by predictor-corrector method. Information Sciences, 2007. 177(7): p. 1633-1647.

16. Nadia, et al., A conformable fractional calculus on arbitrary time scales. Journal of King Saud University Science, 2016.

17. Bhalekar, S., et al., Transient chaos in fractional Bloch equations. Computers \& Mathematics with Applications, 2013. 64(10): p. 3367-3376.

18. elik, V. and Y. Demir, Chaotic Fractional Order Delayed Cellular Neural Network. 2010.

19. Kiani-B, A., et al., A chaotic secure communication scheme using fractional chaotic systems based on an extended fractional Kalman filter. Communications in Nonlinear Science \& Numerical Simulation, 2009. 14(3): p. 863-879.

20. Xu, C., J. Sun, and C. Wang, An Image Encryption Algorithm Based on Random Walk and 
Hyperchaotic Systems. International Journal of Bifurcation and Chaos, 2020.

21. Lan, R., et al., Integrated Chaotic Systems for Image Encryption. Signal Processing, 2018. 147(JUN.): p. 133-145.

22. Penaud, S., et al., DSP implementation of self-synchronised chaotic encoder-decoder. Electronics Letters, 2002. 36(4): p. 365-366.

23. Kharel, R., et al. Implementation of a secure digital chaotic communication scheme on a DSP board. in International Symposium on Communication Systems. 2010.

24. Ye, X., et al., Complexity Analysis of a Mixed Memristive Chaotic Circuit. Complexity, 2018. 2018: p. 1-9.

25. Ye, X., et al., Dynamic Characteristic Analysis for Complexity of Continuous Chaotic Systems Based on the Algorithms of SE Complexity and CO Complexity. 2017.

26. Xiaolin Ye, et al., Analysis of continuous chaotic complexity based on SE and CO algorithm. Journal of Dalian Polytechnic University, 2018. 037(001): p. 67-72. 